... Paul Daverio, Spezialarzt für Plastische und Wiederherstellungs- sowie Mikro-Chirurgie

\title{
«Transsexualität ist ein Identitätsproblem»
}

\section{Daniel Lüthi}

Text und Bilder

danielluethi[at]gmx.ch
In der Szene gilt er als Star, für viele Transsexuelle ist er die grosse Hoffnung. Denn Paul Daverios grosse Spezialität ist es, aus einem Mann eine Frau und aus einer Frau einen Mann zu machen. Vor allem diese zweite Umwandlung hat ihn berühmt gemacht. Denn Daverio kann wie kein anderer Penisse konstruieren.

\section{Umbauten und Neubauten}

Das Haus, in dem Paul Daverio operiert, mutet an wie ein Vier- oder Fünfsternehotel, nicht wie ein Spital. Von der Terrasse aus blicken wir auf den weiten Genfersee und das nahe Frankreich - und in eine medizinische Spezialität, die es mitunter schwer hat, als «normale» medizinische Disziplin anerkannt zu werden. Seit 1986 führt Paul Daverio solche Opera- tionen durch, rund 550 sind es inzwischen geworden. Seine Patientinnen und Patienten kommen aus der ganzen Welt, sie sind zwischen 17 und 63 Jahre alt, im Durchschnitt etwa 30.

Eine Frau zum Mann umzubauen, dauert bei Daverio rund neun Stunden. Er macht es, wie er sagt, «als einziger Chirurg auf der Welt in einem Rutsch», also ohne Unterbruch, in einem Team mit zwei weiteren Chirurgen, zwei Anästhesisten, zwei OP-Schwestern und zwei Pflegenden. Daverio selber ist dabei, so erzählt er, «Urologe, Gefäss- und Neurochirurg, plastischer und ästhetischer Chirurg in einem».

Vorerst einmal baut er eine Brücke zu seinen Vorfahren: «Mein Vater war Architekt, und auch mein Grossvater war im Baugewerbe tätig. Und ich bin

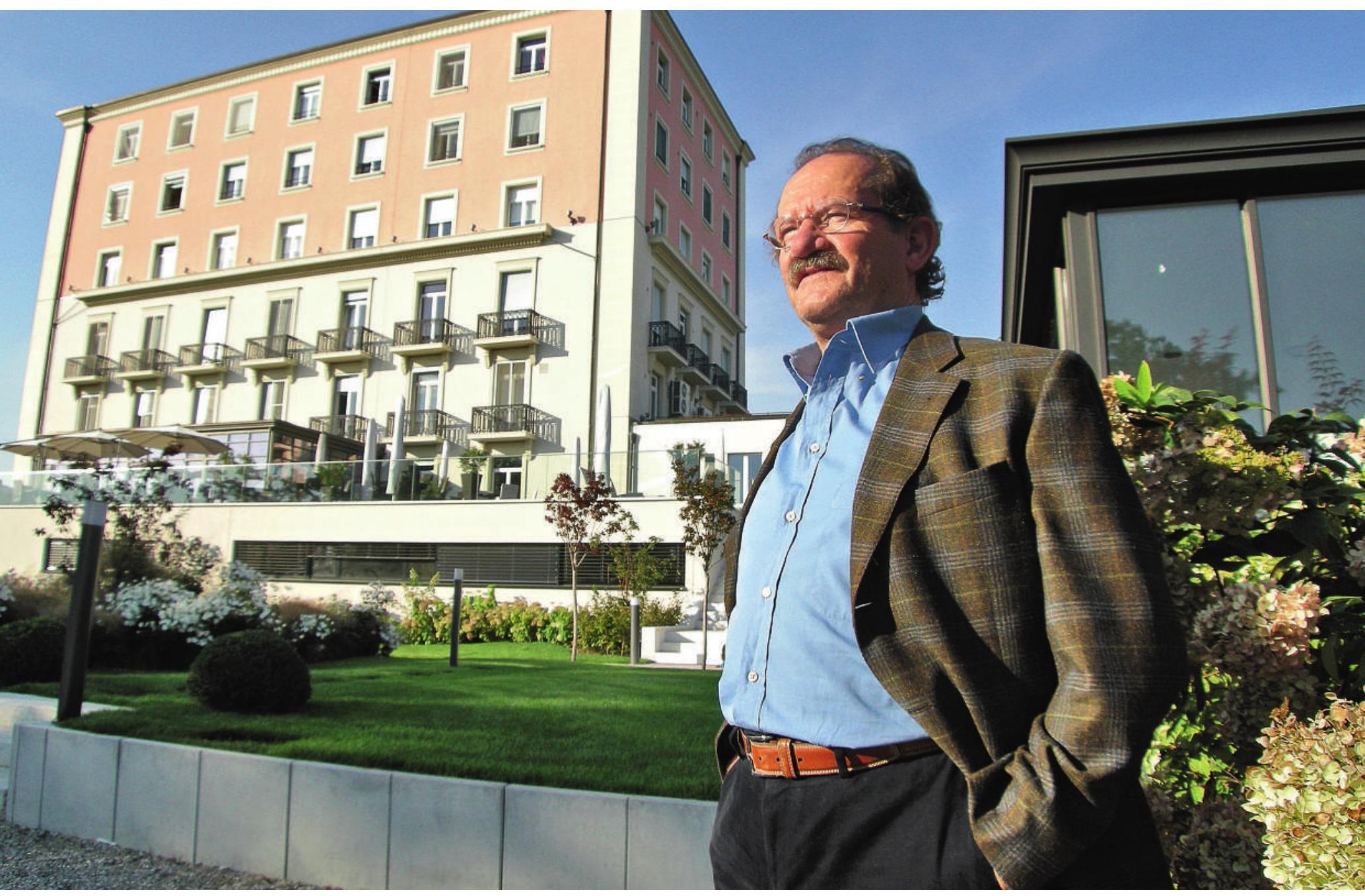


jetzt, in einem anderen Bereich, ebenfalls mit Bauen und Umbauen beschäftigt.»

Dies sind, von Daverio kurz zusammengefasst, die Schritte eines Umbaus von der Frau zum Mann: «Brust weg, Gebärmutter, Eierstöcke und Vagina weg. Harnröhre verlängern beziehungsweise aus den kleinen Schamlippen neu formen, Klitoris verlagern.»

Anschliessend konstruiert er mikrochirurgisch einen Penis: Am Vorderarm der Patientin löst er einen Hautlappen, den er rollt, also zum Phallus formt. Die dazugehörenden Gefässe und Nerven werden abgetragen und später mit den weiblichen Gefässen und Nerven verbunden. Miteinander verbunden werden auch Arterien und Venen, Nerven werden zum Teil geklebt statt genäht - «eine Erfindung von mir». In einem zweiten Eingriff, rund 9 Monate später, wird dann noch der Hodensack fertiggestellt, der aus den ehemaligen grossen Schamlippen gefertigt ist. Darin eingebaut sind Hoden aus Silikon sowie eine kleine Pumpe, mit der die Erektionsprothese betätigt wird.

Was hier beinahe profan tönt, ist für diesen Chirurgen, wie er betont, «immer wieder eine handwerkliche Herausforderung mit einer grossen technischen Faszination». Kaum erstaunlich, dass der gleiche Mann sagt: «Mir war mit 12 schon klar, dass ich Chirurg werden wollte.»

\section{«Transsexualität ist angeboren,}

\section{nicht angeeignet.»}

Stolz ist Daverio heute vor allem auf das geringe Risiko: «Bei gefährlichen Komplikationen wie Thrombosen liegt es unter einem Prozent, das ist wahnsinnig tief, bei ungefährlichen Komplikationen wie Fisteln sind wir bei ungefähr 5 Prozent.»

Aber nicht nur funktionale, sondern auch ästhetische Aspekte spielen für Daverio bei solchen Spezialoperationen eine grosse Rolle: «Ich will ein harmonisches, schönes Resultat, keine Gurke.» Und schliesslich soll die zum Mann gewordene Frau dann ja auch sexuelle Gefühle erleben können: «Die orgastische Sensibilität kommt vom Kern der Klitoris, der im Penis versteckt ist.» Fortpflanzung ist bei der ehemaligen Frau mit dem neu gebauten männlichen Geschlechtsteil nicht möglich, «aber das ist ja bloss ein Problem der Unsterblichkeit.»

Solche fundamentalen Aussagen macht Paul Daverio wie selbstverständlich, begleitet von humoristischer Leichtigkeit oder verhaltenem Sarkasmus. A propos «Fundamentalismus»: Was antwortet der plastische Chirurg jemandem, der ihm vorwirft, er pfusche mit seinen Kreationen dem lieben Gott ins Handwerk? «Nun ja, wenn dies Gottes Sache wäre, hätte er ja schon vor der Geburt eines transsexuellen Menschen aktiv werden können, hätte er den Schal-

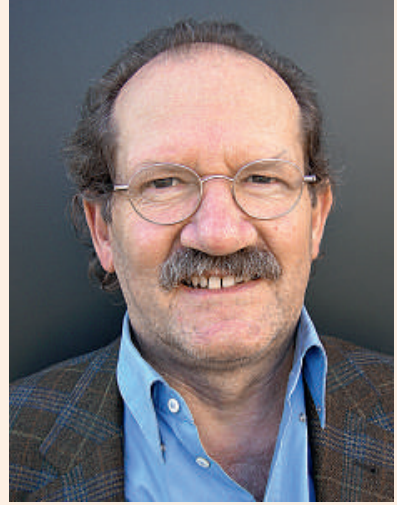

\section{Paul Daverio}

Dr. med. Paul Daverio wurde 1944 im Elsass geboren - als Franzose mit italienischen Wurzeln. Seine Matura machte er in Mulhouse, 1967 kam er in die Schweiz, wo er auch Schweizer wurde. Er studierte Medizin in Mailand, Freiburg und Lausanne. Hier machte er 1973 sein Staatsexamen - und meldete sich gleich für einen Posten an, der erst 2 Jahre später frei werden sollte: eine Assistenzstelle in der Traumatologie des CHUV. In der Zwischenzeit schrieb er Kunstbücher über Tiffanyglas und romantische Bronze. 1975 bis 83 bildete er sich am CHUV in verschiedenen chirurgischen Disziplinen weiter: in der allgemeinen, der Gefäss- und Mikro-, der Neuro-, der HNO- und der Handchirurgie. Eine Pionierleistung in der Schweiz war der Aufbau eines Departements für plastische und Wiederherstellungs-Chirurgie mit dazugehörendem Zentrum für Brandverletzte. 1983 eröffnete Daverio zusammen mit anderen Oberärzten in Lausanne eine eigene Poliklinik, das "Centre Medico-Chirurgical CMC». Hier begann er sich auf die mikrochirurgische Phalloplastik zu spezialisieren. Diese Technik praktiziert er heute vor allem in Lausanne (Klinik Montchoisi) und Potsdam/Deutschland (Klinik Sanssouci), ab und auch in Rom oder Madrid.

Paul Daverio ist verwitwet und Vater von zwei erwachsenen Kindern. Er lebt mit seiner Partnerin, einer Journalistin aus Berlin, in einer Stadtwohnung in Lausanne und auf einem Bauernhof in der Nähe von Divonne in Frankreich. Hier züchtet das Paar arabische Vollblut-Pferde.

ter im Gehirn rechtzeitig in die richtige Richtung betätigen sollen.»

\section{Befreiung aus dem falschen Körper}

Zurück zur Biologie: Was unterscheidet Mann und Frau? «Die Genetik, also die X- und die Y-Chromoso- 
men, und die unterschiedlichen Hormone», sagt Daverio. Und wo ist der biologische «Fehler» oder «Irrtum» zu suchen, von dem im Zusammenhang mit Transsexualität immer wieder die Rede ist? Die Forschung habe Hinweise darauf geliefert, dass sie in Zellen irgendwo im Hypothalamus, also im Gehirn angelegt sei: «Transsexualität ist - wie die Intersexualität übrigens auch - angeboren, nicht angeeignet. Transsexualität ist ein Identitätsproblem», erklärt Paul Daverio. «Anders als andere Chirurgen operieren wir also gesunde, nicht kranke Menschen. Menschen, die im falschen Körper eingesperrt sind. Ich kann sie mit meiner Operation befreien. Viele meiner Patientinnen und Patienten sagen als erstes, wenn sie aus der Narkose erwachen, denn auch: ‘Dies ist mein eigentlicher Geburtstag.> Aber: Die eigentliche Umwandlung machen die Hormone, nicht wir Chirurgen. Unsere Arbeit ist nur die Vollendung, die Kirsche auf der Torte.»

\section{Ästhetische und andere Medizin}

$\mathrm{Ab}$ und $\mathrm{zu}$ verschönert dieser kleingewachsene, umtriebige Mann auch mal eine Nase, eine Brust oder ein Kinn. Er macht ein Lifting oder glättet mittels Botox eine Falte: «Dies ist jedoch nicht Medizin, sondern Kosmetik - ein kleiner Service nebenbei, eine kleine Dienstleistung wie diejenige eines Friseurs.» Verwerflich oder gar pervers finde er derlei Schönheitschirurgie nicht, auch wenn einer damit viel Geld verdienen könne: «Was ist schon pervers?», fragt Daverio zurück, «die ganze Welt ist pervers, übertreiben ist pervers. Einmal zum Beispiel habe ich im Sinn einer humanitären Hilfeleistung Soldaten aus Kriegsgebieten zusammengeflickt. Nach erfolgreicher Operation mit umfangreichen Hauttransplantationen gingen sie zurück - um sich wieder zusammenschiessen zu lassen. Das ist doch pervers, oder nicht?»

Wenn Paul Daverio von seinem zweiten Wohnsitz jenseits der Schweizer Grenze erzählt, leuchten

\section{«Ich heile Seelen, denn die Menschen, die ich operiere, sind vorher unglücklich und nachher glücklich.»}

Die wundersame Wandlung beginnt also schon lange vor den Aktionen auf dem Operationstisch: mit der eigenen Auseinandersetzung und mit der Sensibilisierung des persönlichen Umfeldes, der Aufklärung von Verwandten und Bekannten also. Hausärzte und Psychiater sollten in diesem Prozess eine wichtige Rolle spielen, erklärt Daverio in seiner gewohnt entspannte aber präsenten und prägnanten Art, «aber viele Ärzte haben leider keine Ahnung von dieser Problematik.» Nun ja, das Phänomen der Transsexualität ist relativ selten: auf 40000 Geburten kommt ein Fall.

$\mathrm{Zu} 80$ Prozent macht Paul Daverio aus Frauen Männer. Eine solche Umwandlung kostet rund 100000 Franken - «alles inbegriffen», wie er betont, also ärztliche Honorare, Klinik, Pflege und Nachbehandlung. Und zu 20 Prozent macht er aus Männern Frauen: «Dieser Umbau ist nur halb so kompliziert, dauert nur halb so lang und kostet deshalb nur etwa halb so viel.»

Bei Privatpatientinnen und -patienten bezahle die Krankenkasse. seine Augen. Auf einem Bauernhof in Frankreich züchtet er zusammen mit seiner Partnerin Pferde, «arabisches Vollblut», wie er mit sichtbarem Stolz beifügt, «zurzeit sind nur noch etwa 30 Stück auf dem Hof.»

Wir kommen auf die Kinder zu sprechen: den Sohn, der Kunstmaler ist, und die Tochter, die Ernährungswissenschaft studiert hat.

Und plötzlich sind wir in unserem Gespräch bei Daverios verstorbener Frau. Vor drei Jahren starb sie an der seltenen und unheilbaren Nervenkrankheit ALS.

Jetzt sind wir gedanklich auf einmal in einer völlig anderen medizinischen Welt: «Ja, für einen Arzt wie mich ist es besonders schwierig, so etwas so hautnah miterleben zu müssen.»

Wäre nicht das seine eigentliche Berufung: heilen und Leben retten? «Nicht jeder Arzt kann Leben retten», sinniert Daverio auf der Terrasse dieser Klinik, die mehr wie ein Fünfsternhotel anmutet als wie ein Spital. «Ich tue auch Gutes: Ich heile Seelen. Denn die Menschen, die ich operiere, sind vorher unglücklich, und nachher glücklich.»

\section{Die nächste «Begegnung mit ...»}

Am Ende jeden Monats stellt die Schweizerische Ärztezeitung eine Persönlichkeit vor, die sich im Gesundheitswesen engagiert. Im Dezember schildert Daniel Lüthi seine Begegnung mit Marianne Indergand-Erni, frei praktizierende und Beleg-Hebamme in Stans. 